\title{
Multiscale Discontinuous Galerkin Methods for Modeling Flow and Transport in Porous Media
}

\author{
Shuyu Sun ${ }^{1}$ and Jürgen Geiser ${ }^{2}$ \\ ${ }^{1}$ Department of Mathematical Sciences, Clemson University \\ O-221 Martin Hall, Clemson, SC 29634-0975, USA \\ shuyu@clemson.edu \\ 2 Department of Mathematics, Humboldt-Universität zu Berlin \\ Unter den Linden 6, D-10099 Berlin, Germany \\ geiser@mathematik.hu-berlin.de
}

\begin{abstract}
Multiscale discontinuous Galerkin (DG) methods are established to solve flow and transport problems in porous media. The underlying idea is to construct local DG basis functions at the coarse scale that capture the local properties of the differential operator at the fine scale, and then to solve the DG formulation using the newly constructed local basis functions instead of conventional polynomial functions on the coarse scale elements. Numerical examples are provided for demonstrating their effectiveness.
\end{abstract}

\section{Introduction}

Flow and reactive transport in porous media are fundamental processes arising in many diversified fields such as petroleum engineering, groundwater hydrology, environmental engineering, soil mechanics, earth sciences, chemical engineering and biomedical engineering [13,8. Major challenges for realistic simulations of simultaneous flow, transport and chemical reaction include multiple temporal and spatial scales, long simulation time periods, and multiple coupled nonlinear components. In particular, the critical effect of fine scale processes on coarser scales requires numerical methods to effectively account for the interactions across various scales. Since it is far beyond current computational power to resolve the finest scale directly, one usually has to incorporate the fine scale effects within a coarse-scale approximation by various multiscale numerical schemes [4,5].

DG are finite element methods using discontinuous piecewise polynomial spaces and specialized bilinear forms to weakly impose boundary conditions and interelement continuities [6, 7, 1, 11,3]. The methods have recently become popular in the scientific and engineering communities due to their many appealing features including local mass conservation, small numerical diffusion and little oscillation, and support of variable local approximations and nonconforming meshes. In this paper, we shall establish a two-scale framework for primal DG methods. 


\section{Discontinuous Galerkin Schemes}

\subsection{Governing Equations}

Let $\Omega$ denote a bounded polygonal domain in $\mathbb{R}^{d},(d=1,2$, or 3$)$ and let $T$ denote the final simulation time. The flow problem we consider is single-phase flow in porous media:

$$
-\nabla \cdot(\mathbf{K} \nabla p) \equiv \nabla \cdot \mathbf{u}=q, \quad(x, t) \in \Omega \times(0, T],
$$

where the pressure $p$ or the Darcy's velocity is the unknown variable to be solved. The conductivity $\mathbf{K}$ is a given parameter, and it usually varies greatly with geological space. The imposed external total flow rate $q$ is a sum of sources (injection) and sinks (extraction).

The modeling equation for single-species reactive transport in a single flowing phase in porous media involves both spatial and temporal derivatives:

$$
\frac{\partial \phi c}{\partial t}+\nabla \cdot(\mathbf{u} c-\mathbf{D}(\mathbf{u}) \nabla c)=q c^{*}+r(c),(x, t) \in \Omega \times(0, T],
$$

where the species concentration $c$ is the unknown variable to be solved. The effective porosity $\phi$ is a given parameter, and it usually varies spatially. The dispersiondiffusion tensor $\mathbf{D}(\mathbf{u})$ is a given function of Darcy velocity $\mathbf{u}$. The notation $c^{*}$ is the injected concentration $c_{w}$ if $q>0$ and is the resident concentration $c$ if $q \leq 0$. The reaction term $r(c)$ is a given function to describe the kinetic biogeochemical reaction.

We write $\partial \Omega=\bar{\Gamma}_{D} \cup \bar{\Gamma}_{N}=\bar{\Gamma}_{\text {in }} \cup \bar{\Gamma}_{\text {out }}$, where $\Gamma_{D}$ is the Dirichlet boundary and $\Gamma_{N}$ is the Neumann boundary for the flow subproblem and $\Gamma_{D} \cap \Gamma_{N}=\emptyset$; $\Gamma_{\text {in }}$ is the inflow boundary and $\Gamma_{\text {out }}$ is the outflow/noflow boundary condition. We assume the following boundary conditions:

$$
\begin{aligned}
& p=p_{B}, \quad(x, t) \in \Gamma_{D} \times(0, T], \\
& \mathbf{u} \cdot \mathbf{n}=u_{B}, \quad(x, t) \in \Gamma_{N} \times(0, T], \\
& (\mathbf{u} c-\mathbf{D}(\mathbf{u}) \nabla c) \cdot \mathbf{n}=c_{B} \mathbf{u} \cdot \mathbf{n}, \quad(x, t) \in \Gamma_{\mathrm{in}} \times(0, T], \\
& (-\mathbf{D}(\mathbf{u}) \nabla c) \cdot \mathbf{n}=0, \quad(x, t) \in \Gamma_{\text {out }} \times(0, T] .
\end{aligned}
$$

The initial condition is specified by

$$
c(x, 0)=c_{0}(x), \quad x \in \Omega .
$$

Here, $c_{B}$ is the inflow boundary concentration and $c_{0}$ the initial concentration.

\subsection{DG Schemes}

Let $\mathcal{E}_{h}$ be a family of non-degenerate and possibly non-conforming partitions of $\Omega$ composed of line segments if $d=1$, triangles or quadrilaterals if $d=2$, or tetrahedra, prisms or hexahedra if $d=3$. The set of all interior points (for $d=1$ ), edges (for $d=2$ ), or faces (for $d=3$ ) for $\mathcal{E}_{h}$ is denoted by $\Gamma_{h}$. The discontinuous finite 
element space is taken to be $\mathcal{D}_{r}\left(\mathcal{E}_{h}\right):=\left\{\phi \in L^{2}(\Omega):\left.\phi\right|_{E} \in \mathbb{P}_{r}(E), E \in \mathcal{E}_{h}\right\}$, where $\mathbb{P}_{r}(E)$ denotes the space of polynomials of (total) degree less than or equal to $r$ on $E$. Let $E_{i}, E_{j} \in \mathcal{E}_{h}$ and $\gamma=\partial E_{i} \cap \partial E_{j} \in \Gamma_{h}$ with $\mathbf{n}_{\gamma}$ exterior to $E_{i}$. We now define the average and jump for a smooth function $\phi$ by $\{\phi\}:=\frac{1}{2}\left(\left.\left(\left.\phi\right|_{E_{i}}\right)\right|_{\gamma}+\left.\left(\left.\phi\right|_{E_{j}}\right)\right|_{\gamma}\right),[\phi]:=\left.\left(\left.\phi\right|_{E_{i}}\right)\right|_{\gamma}-\left.\left(\left.\phi\right|_{E_{j}}\right)\right|_{\gamma}$. Denote the upwind value of the concentration $\left.c^{*}\right|_{\gamma}$ as $\left.c\right|_{E_{i}}$ if $\mathbf{u} \cdot \mathbf{n}_{\gamma} \geq 0$ and $\left.c\right|_{E_{j}}$ if $\mathbf{u} \cdot \mathbf{n}_{\gamma}<0$. We introduce the bilinear forms $a(p, \psi)$ and $B(c, w ; \mathbf{u})$ for the flow and transport problems respectively:

$$
\begin{aligned}
a(p, \psi):= & \sum_{E \in \mathcal{E}_{h}} \int_{E} \mathbf{K} \nabla p \cdot \nabla \psi+J_{0}^{\sigma}(p, \psi) \\
& -\sum_{\gamma \in \Gamma_{h}} \int_{\gamma}\left\{\mathbf{K} \nabla p \cdot \mathbf{n}_{\gamma}\right\}[\psi]-s_{\text {form }} \sum_{\gamma \in \Gamma_{h}} \int_{\gamma}\left\{\mathbf{K} \nabla \psi \cdot \mathbf{n}_{\gamma}\right\}[p]+ \\
& -\sum_{\gamma \subset \Gamma_{D}} \int_{\gamma} K \nabla p \cdot \mathbf{n}_{\gamma} \psi-s_{\text {form }} \sum_{\gamma \subset \Gamma_{D}} \int_{\gamma} K \nabla \psi \cdot \mathbf{n}_{\gamma} p, \\
B(c, w ; \mathbf{u}):= & \sum_{E \in \mathcal{E}_{h}} \int_{E}(\mathbf{D}(\mathbf{u}) \nabla c-c \mathbf{u}) \cdot \nabla w-\int_{\Omega} c q^{-} w \\
& -\sum_{\gamma \in \Gamma_{h}} \int_{\gamma}\left\{\mathbf{D}(\mathbf{u}) \nabla c \cdot \mathbf{n}_{\gamma}\right\}[w]-s_{\text {form }} \sum_{\gamma \in \Gamma_{h}} \int_{\gamma}\left\{\mathbf{D}(\mathbf{u}) \nabla w \cdot \mathbf{n}_{\gamma}\right\}[c] \\
& +\sum_{\gamma \in \Gamma_{h}} \int_{\gamma} c^{*} \mathbf{u} \cdot \mathbf{n}_{\gamma}[w]+\sum_{\gamma \subset \Gamma_{\mathrm{out}}} \int_{\gamma} c \mathbf{u} \cdot \mathbf{n}_{\gamma} w+J_{0}^{\sigma}(c, w),
\end{aligned}
$$

where $s_{\text {form }}=-1$ for NIPG (the Nonsymmetric Interior Penalty Galerkin method 7]) or OBB-DG (the Oden-Babuška-Baumann formulation of DG [6]), $s_{\text {form }}=1$ for SIPG (the Symmetric Interior Penalty Galerkin method [12,9.11]), and $s_{\text {form }}=$ 0 for IIPG (the Incomplete Interior Penalty Galerkin method [9, 2, 11, 10]). For notational convenience, we use the same $s_{\text {form }}$ and penalty term for both flow and transport problems, but they can be chosen differently in practice. Here $q^{+}$is the injection source term, and $q^{-}$is the extraction source term, i.e., $q^{+}:=\max (q, 0)$, $q^{-}:=\min (q, 0)$. In addition, we define the interior penalty term as $J_{0}^{\sigma}(c, w):=$ $\sum_{\gamma \in \Gamma_{h}} r^{2} \sigma_{\gamma} \int_{\gamma}[c][w] / h_{\gamma}$, where $\sigma_{\gamma}$ is the penalty parameter on $\gamma$. We have $\sigma_{\gamma} \equiv 0$ for OBB-DG. The linear functionals for flow and transport problems are defined respectively as

$$
\begin{aligned}
l(\psi) & :=(q, \psi)-s_{\text {form }} \sum_{\gamma \subset \Gamma_{D}} \int_{e} K \nabla \psi \cdot \mathbf{n}_{\gamma} p_{B}-\sum_{\gamma \subset \Gamma_{N}} \int_{e} \psi u_{B}, \\
L(w ; \mathbf{u}, c) & :=\int_{\Omega} r(c) w+\int_{\Omega} c_{w} q^{+} w-\sum_{\gamma \subset \Gamma_{\mathrm{in}}} \int_{\gamma} c_{B} \mathbf{u} \cdot \mathbf{n}_{\gamma} w .
\end{aligned}
$$

For the flow problem (10) and (3)-(4), we seek a time-independent solution $P^{D G} \in \mathcal{D}_{r}\left(\mathcal{E}_{h}\right)$ satisfying,

$$
a\left(P^{D G}, v\right)=l(v), \quad \forall v \in \mathcal{D}_{r}\left(\mathcal{E}_{h}\right) .
$$


For the transport problem (2) and (5)-(7), the continuous-in-time DG approximation $C^{D G}(\cdot, t) \in \mathcal{D}_{r}\left(\mathcal{E}_{h}\right)$ is the solution of the following ODEs:

$$
\begin{aligned}
\left(\frac{\partial \phi C^{D G}}{\partial t}, w\right)+B\left(C^{D G}, w ; \mathbf{u}^{D G}\right) & =L\left(w ; \mathbf{u}^{D G}, C^{D G}\right) \\
\forall w & \in \mathcal{D}_{r}\left(\mathcal{E}_{h}\right), \quad t \in(0, T] \\
\left(\phi C^{D G}, w\right) & =\left(\phi c_{0}, w\right), \quad \forall w \in \mathcal{D}_{r}\left(\mathcal{E}_{h}\right), t=0 .
\end{aligned}
$$

\section{Multiscale Formulation for Discontinuous Galerkin}

We now establish a multiscale DG formulation for solving the flow problem in porous media. The same multiscale approach can be applied to the transport problem as well, but is omitted here for brevity. In a fine mesh $\mathcal{E}_{h}$, we consider an $r$-order approximation space $\mathcal{D}_{r}\left(\mathcal{E}_{h}\right)$. The DG solution $P_{h}$ in the space $\mathcal{D}_{r}\left(\mathcal{E}_{h}\right)$ can be obtained by solving the following algebraic linear system:

$$
a\left(P_{h}, v\right)=l(v), \quad \forall v \in \mathcal{D}_{r}\left(\mathcal{E}_{h}\right) .
$$

Flow in geological media usually involves behaviors across several scales, but direction solution at a very fine scale is far beyond current computational power. Let us assume that we can only afford to solve the flow equation in a coarse space $\mathcal{D}_{R}\left(\mathcal{E}_{H}\right)$ directly, where $R<r$ and $H>h$. That is, current computational capability only allows us to solve for $P_{H}$ in the space $\mathcal{D}_{R}\left(\mathcal{E}_{H}\right)$ from the following algebraic system:

$$
a\left(P_{H}, v\right)=l(v), \quad \forall v \in \mathcal{D}_{R}\left(\mathcal{E}_{H}\right) .
$$

We note that the bilinear form $a(\cdot, \cdot)$ contains slightly different terms on different meshes. However, by choosing the penalty parameters properly, we can make the coarse mesh bilinear form identical to the restriction of the fine mesh bilinear form to the coarse space.

Since the conductivity (or permeability) could be highly oscillatory on the fine scale, we would like to incorporate the fine scale effects within a coarse-scale approximation. One natural solution is to decompose the fine space into the coarse space component and its complement: $\mathcal{D}_{r}\left(\mathcal{E}_{h}\right)=\mathcal{D}_{R}\left(\mathcal{E}_{H}\right) \oplus V_{f}$. If we seek $P_{H} \in \mathcal{D}_{R}\left(\mathcal{E}_{H}\right)$ and $P_{f} \in V_{f}$ such that

$$
\begin{aligned}
a\left(P_{H}, v_{H}\right) & =l\left(v_{H}\right)-a\left(P_{f}, v_{H}\right), & & \forall v_{H} \in \mathcal{D}_{R}\left(\mathcal{E}_{H}\right), \\
a\left(P_{f}, v_{f}\right) & =l\left(v_{f}\right)-a\left(P_{H}, v_{f}\right), & & \forall v_{f} \in V_{f} .
\end{aligned}
$$

We can recover the solution $P_{h}$ at the fine scale by letting $P_{h}=P_{H}+P_{f}$. Giving $P_{f}$, (14) requires only computation of a solution in the coarse space, and thus is affordable. However, (15) is still computationally intractable. To 
improve efficiency, we replace the space $V_{f}$ by a locally supported space: $V_{f 0}:=$ $\left\{v \in V_{f}:\left.v\right|_{\partial E}=0, \forall E \in \mathcal{E}_{H}\right\}$. Our multiscale algorithm is to seek $P_{H} \in \mathcal{D}_{R}\left(\mathcal{E}_{H}\right)$ and $P_{f 0} \in V_{f 0}$ such that

$$
\begin{aligned}
a\left(P_{H}, v_{H}\right) & =l\left(v_{H}\right)-a\left(P_{f 0}, v_{H}\right), & & \forall v_{H} \in \mathcal{D}_{R}\left(\mathcal{E}_{H}\right), \\
a\left(P_{f 0}, v_{f 0}\right) & =l\left(v_{f 0}\right)-a\left(P_{H}, v_{f 0}\right), & & \forall v_{f 0} \in V_{f 0} .
\end{aligned}
$$

We note that (17) is decoupled among the elements in the coarse mesh $\mathcal{E}_{H}$. To solve (17), it is equivalent to solve $N$ local problems, where $N$ is the number of elements in the coarse mesh $\mathcal{E}_{H}$. For each element $E \in \mathcal{E}_{H}$, the local problem is to seek $\left.P_{f 0, E} \in V_{f 0}\right|_{E}$ such that

$$
a\left(P_{f 0, E}, v_{f 0, E}\right)=l\left(v_{f 0, E}\right)-a\left(P_{H}, v_{f 0, E}\right),\left.\quad \forall v_{f 0, E} \in V_{f 0}\right|_{E} .
$$

The final solution we obtain is $P_{\mathrm{MS}}:=P_{H}+P_{f 0}=P_{H}+\sum_{E \in \mathcal{E}_{H}} P_{f 0, E}$.

The two equations (16) and (17) are coupled through the right-hand sides. Though we can solve the two-scale system (16)-(17) iteratively, but a substitution method is more efficient. For each basis function $v_{H}$ in the coarse space $V_{H}$, we solve for $\phi\left(v_{H}\right) \in V_{f 0}$ such that

$$
a\left(\phi\left(v_{H}\right), v_{f 0}\right)=l\left(v_{f 0}\right)-a\left(v_{H}, v_{f 0}\right), \quad \forall v_{f 0} \in V_{f 0} .
$$

Of course, the solution of $\phi\left(v_{H}\right)$ involves only a local problem within a coarse mesh element. We then construct the multiscale basis by $\phi_{\mathrm{MS}}\left(v_{H}\right):=\phi\left(v_{H}\right)+v_{H}$. Obviously, we know $a\left(\phi_{\mathrm{MS}}\left(v_{H}\right), v_{f 0}\right)=l\left(v_{f 0}\right), \forall v_{f 0} \in V_{f 0}$. We now define a multiscale approximation space $V_{\mathrm{MS}}:=\operatorname{span}\left\{\phi_{\mathrm{MS}}\left(v_{H}\right): \forall v_{H} \in \mathcal{D}_{R}\left(\mathcal{E}_{H}\right)\right\}$. Now our multiscale scheme (16)-(17) becomes the problem of seeking $P_{\mathrm{MS}} \in V_{\mathrm{MS}}$ such that

$$
a\left(P_{\mathrm{MS}}, v_{\mathrm{MS}}\right)=l\left(v_{\mathrm{MS}}\right), \quad \forall v_{\mathrm{MS}} \in V_{\mathrm{MS}} .
$$

Since the multiscale space $V_{\mathrm{MS}}$ has the same dimension as the coarse space $\mathcal{D}_{R}\left(\mathcal{E}_{H}\right)$, the computational time and memory requirement for solving (18) is similar to that for solving (13), provided the multiscale basis functions have been constructed. The construction of multiscale basis involves only solutions of local problems and can be obtained efficiently. In addition, the problem coefficient for the construction of multiscale basis does not have to be stored, which substantially saving memory requirement compared to the direction solution of the flow problem in the fine space.

\section{Numerical Results}

We consider the problem (11)-(17) over the domain $\Omega=(0,1)^{2}$. The conductivity tensor $\mathbf{K}$ is a diagonal matrix with $\mathbf{K}_{11}=\mathbf{K}_{22}$, and the distribution of $\mathbf{K}_{11}$ across the domain is plotted in Figure 1. We impose no source/sink term, i.e. $q=0$. For the flow problem, we impose Dirichlet boundary conditions $p=1$ and $p=0$ on the left and right boundaries, respectively. The boundary conditions 


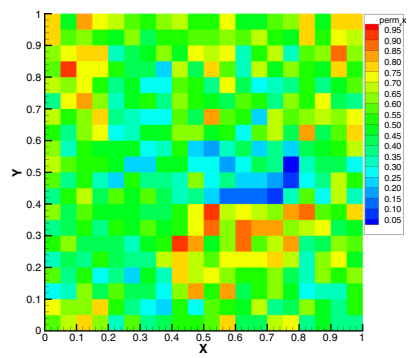

Fig. 1. Conductivity field for the flow problem

on top and bottom boundaries are assumed to be no-flow. For the transport problem, we let the effective porosity to be 1 (for convenience), we impose no reaction and we assume zero diffusion-dispersion. The advection part uses the Darcy velocity computed from the flow problem, with inflow concentration being constant 1 and with initial concentration being 0 everywhere.

NIPG is employed to solve the flow problem. The penalty parameter for NIPG is chosen to be 1 . The fine mesh used is a 20x20 uniform rectangular grid and the coarse one is a $5 \times 5$ uniform rectangular mesh. The polynomial degree for the flow problem is $R=r=2$ for both the fine and the coarse spaces. For the transport problem, we only test the Darcy velocity computed from flow for predicting advection behaviors, and thus we use fine mesh for all cases. Advection is solved by OBB-DG with lowest order polynomials (constants) and explicit Euler's temporal integration with uniform time step $\Delta t=0.001$. The spatial discretization for advection here is equivalent to the upwinding finite volume method.

Figure 2 displays the results of pressure, velocity and concentration. The results from our multiscale algorithm are plotted in the first column. For comparison, the DG results in the coarse and fine meshes are provided in the second and third columns. Comparison indicates the computed pressure from the multiscale approach is very close to the one computed from DG in the fine mesh. Unlike the coarse DG result, the multiscale pressure result captures the fine scale information in a similar fashion as the DG result in the fine mesh; this is especially pronounced in the area around the point $(0.8,0.5)$. Simulations of advection indicate that the multiscale DG and coarse mesh DG methods produce nonphysical overshoots and undershoots, while the fine mesh DG method possesses no overshoot/undershoot. This is because the velocity produced from the fine mesh DG method is locally mass conservative in the fine mesh but the velocities computed from the multiscale DG and coarse mesh DG methods are not. However, the overshoot/undershoot produced by the multiscale DG is much smaller than that produced by the coarse mesh DG method. We also note that the multiscale approach leads to inaccurate velocity in a few locations along the no-flow boundary, particularly at the point $(0.1,1)$. This is because we strongly impose zero pressure in the local problem for computing multiscale basis functions. A potential improvement is to collect the local problems that touch the 
domain no-flow boundary and modify the corresponding boundaries in the local problems by no-flow rather than zero Dirichlet boundary condition.

Compared to the classical multiscale implementation of continuous Galerkin methods 4, the multiscale DG scheme is computational simpler. Unlike continuous Galerkin, the support of coarse basis function in DG sits within a single coarse mesh element, thus the construction of multiscale basis function is also limited to a single coarse mesh element. In addition, the multiscale DG algorithm has more flexibility in the construction of multiscale basis functions. For example, in this paper, we have strongly enforced zero Dirichlet boundary condition for the local problems to construct multiscale basis functions. However, we could also use other boundary conditions in implementation. For example, we could weakly impose homogeneous Dirichlet boundary condition for the local problems, or we could strongly or weakly impose homogeneous Neumann (i.e. no-flow) boundary condition for the local problems.
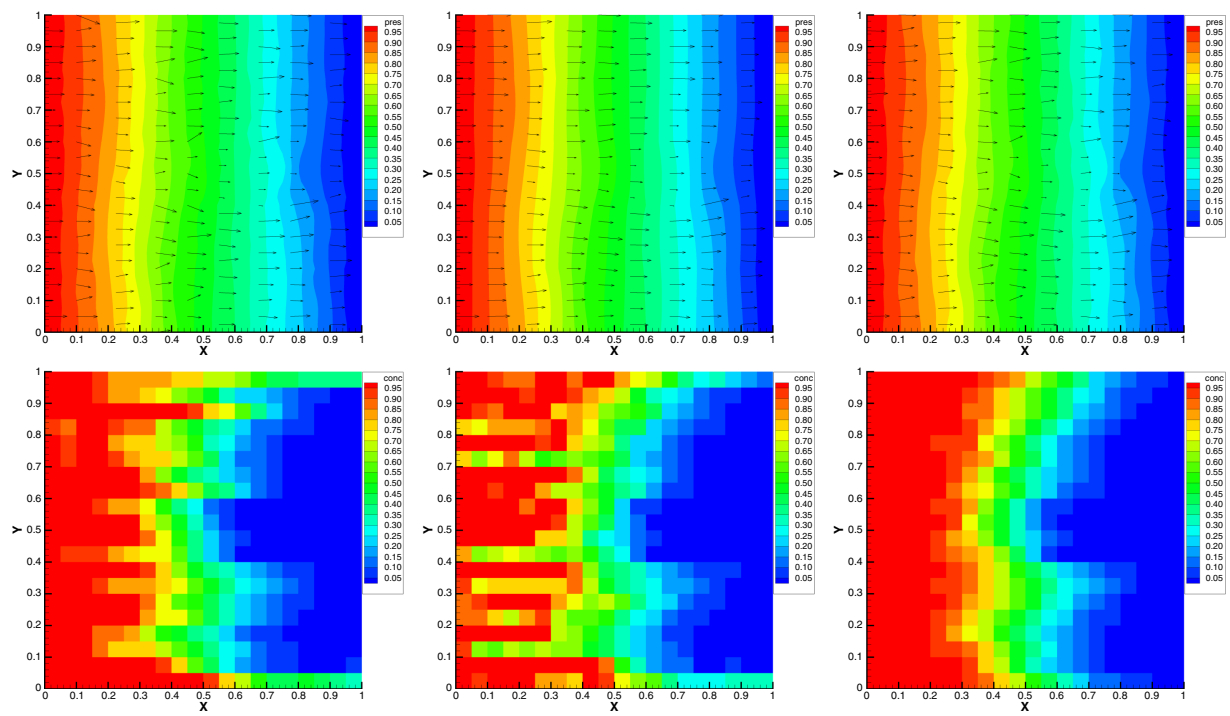

Fig. 2. NIPG results (left column: multiscale DG solution; middle column: coarse mesh DG solution; right column: fine mesh DG solution; top row: pressure and velocity for the flow problem; bottom row: concentration at $t=1$ for the transport problem)

\section{Conclusions}

The four primal DG schemes, namely, NIPG, OBB-DG, SIPG, and IIPG, have been considered to solve flow and transport problems in porous media. We have systematically establish a theoretical framework for multiscale DG methods. The underlying idea is first to construct local basis functions at the coarse scale that capture the local properties of the differential operator at the fine scale. The 
second step of the algorithm is to solve the DG formulation using the newly constructed local basis functions instead of conventional polynomial functions on the coarse scale elements. Numerical examples have been provided to demonstrate that the proposed multiscale DG indeed captures many important features of flow and transport at fine scales but yet cost roughly only the computational time of the corresponding coarse scale problem. We have also compared multiscale DG algorithms with multiscale continuous Galerkin methods, and found that the multiscale implementation of DG is probably simpler and more flexible than the multiscale implementation of continuous Galerkin. A future work is to investigate various other options for multiscale DG implementation; in particular, it will be interesting to weakly impose homogeneous Neumann condition for the local problems to construct multiscale basis functions, as this will likely reduce the error of the velocity and introduce little nonphysical oscillation.

\section{References}

1. Arnold, D. N.: An interior penalty finite element method with discontinuous elements. SIAM J. Numer. Anal. 19 (1982) 742-760

2. Dawson, C., Sun, S., Wheeler, M. F.: Compatible algorithms for coupled flow and transport. Comput. Meth. Appl. Mech. Eng. 193 (2004) 2565-2580

3. Geiser, J.: Mixed discretisation methods for the discontinuous galerkin method with analytical test-functions. Preprint No. 2006-8 of Humboldt University of Berlin, Department of Mathematics, Germany (2006)

4. Hou, T. Y., Wu, X.: A multiscale finite element method for elliptic problems in composite materials and porous media. J. Comput. Phys. 134 (1997) 169-189

5. Hughes, T. J. R.: Multiscale phenomena: Green's functions, the Dirichlet-toNeumann formulation, subgrid scale models, bubbles and the origins of stabilized methods. Comput. Methods Appl. Mech. Engrg. 127 (1995) 387-401

6. Oden, J. T., Babuška, I., Baumann, C. E.: A discontinuous $h p$ finite element method for diffusion problems. J. Comput. Phys. 146 (1998) 491-516

7. Rivière, B., Wheeler, M. F., Girault, V.: A priori error estimates for finite element methods based on discontinuous approximation spaces for elliptic problems. SIAM J. Numer. Anal. 39 (2001) 902-931

8. Steefel, C. I., Van Cappellen, P.: Special issue: Reactive transport modeling of natural systems. Journal of Hydrology. 209 (1998) 1-388

9. Sun, S.: Discontinuous Galerkin methods for reactive transport in porous media. $\mathrm{PhD}$ thesis, The University of Texas at Austin (2003)

10. Sun, S., Wheeler, M. F.: Discontinuous Galerkin methods for coupled flow and reactive transport problems. Appl. Numer. Math. 52 (2005) 273-298

11. Sun, S., Wheeler, M. F.: Symmetric and nonsymmetric discontinuous Galerkin methods for reactive transport in porous media. SIAM Journal on Numerical Analysis. 43 (2005) 195-219

12. Wheeler,M. F.: An elliptic collocation-finite element method with interior penalties. SIAM J. Numer. Anal. 15 (1978) 152-161

13. Yeh, G. T., Tripathi, V. S.: A model for simulating transport of reactive multispecies components: model development and demonstration. Water Resources Research. 27 (1991) 3075-3094 